\title{
Affine Weyl Group Symmetry of the Garnier System
}

\author{
By
}

\author{
Takao SUZUKI
}

(Kobe University, Japan)

\begin{abstract}
In this paper, we show that the Garnier system in $n$-variables has affine Weyl group symmetry of type $B_{n+3}^{(1)}$. We also formulate the $\tau$-functions for the Garnier system (or the Schlesinger system of rank 2) on the root lattice $Q\left(C_{n+3}\right)$ and show that they satisfy Toda equations, Hirota-Miwa equations and bilinear differential equations.

Keywords and Phrases. Affine Weyl group, Bäcklund transformations, Garnier system, Schlesinger system, $\tau$-functions.

2000 Mathematics Subject Classification Numbers. 33E17, 20F55, 37K35.
\end{abstract}

\section{Introduction}

For the sixth Painlevé equation $P_{V I}$, the symmetry structure is well-known $[1,5]$. Furthermore, the $\tau$-functions for $P_{V I}$ satisfy various bilinear relations [4, $5,6]$. But such properties are not clarified completely for the Garnier system which is an extension of $P_{V I}$ to several variables. In this paper, we show that the Garnier system in $n$-variables $(n \geq 2)$ has affine Weyl group symmetry of type $B_{n+3}^{(1)}$. We also formulate the $\tau$-functions for the Garnier system (or the Schlesinger system of rank 2) on the root lattice $Q\left(C_{n+3}\right)$ and show that they satisfy Toda equations, Hirota-Miwa equations and bilinear differential equations.

Consider a Fuchsian differential equation on $\boldsymbol{P}^{1}(\boldsymbol{C})$

$$
\frac{d^{2} y}{d z^{2}}+P_{1}(z) \frac{d y}{d z}+P_{2}(z) y=0
$$

with regular singularities $z=t_{1}, \ldots, t_{n}, t_{n+1}=0, t_{n+2}=1, t_{n+3}=\infty$, apparent singularities $z=\lambda_{1}, \ldots, \lambda_{n}$ and the Riemann scheme

$$
\left(\begin{array}{ccccccc}
z=t_{1} & \cdots & z=t_{n+2} & z=t_{n+3} & z=\lambda_{1} & \cdots & z=\lambda_{n} \\
0 & \cdots & 0 & \rho & 0 & \cdots & 0 \\
\theta_{1} & \cdots & \theta_{n+2} & \rho+\theta_{n+3}+1 & 2 & \cdots & 2
\end{array}\right)
$$

assuming that the Fuchs relation 


$$
\sum_{j=1}^{n+3} \theta_{j}+2 \rho=0
$$

is satisfied. The monodromy preserving deformations of the equation (1.1) with the scheme (1.2) is described as the following completely integrable Hamiltonian system [1]:

$$
\frac{\partial \lambda_{j}}{\partial t_{i}}=\frac{\partial \mathscr{K}_{i}}{\partial \mu_{j}}, \quad \frac{\partial \mu_{j}}{\partial t_{i}}=-\frac{\partial \mathscr{K}_{i}}{\partial \lambda_{j}} \quad(i, j=1, \ldots, n),
$$

where

$$
\mu_{j}=\operatorname{Res}_{z=\lambda_{j}} P_{2}(z) d z \quad(j=1, \ldots, n)
$$

and $\mathscr{K}_{i}(i=1, \ldots, n)$ are rational functions in $\lambda_{j}, \mu_{j}(j=1, \ldots, n)$ given by

$$
\mathscr{K}_{i}=-\operatorname{Res}_{z=t_{i}} P_{2}(z) d z .
$$

By the canonical transformation

$$
x_{i}=\frac{t_{i}}{t_{i}-1}, \quad q_{i}=\frac{t_{i} \prod_{j=1}^{n}\left(t_{i}-\lambda_{j}\right)}{\prod_{j=1, j \neq i}^{n+2}\left(t_{i}-t_{j}\right)} \quad(i=1, \ldots, n),
$$

the system (1.4) is transformed into the Hamiltonian system

$$
\frac{\partial q_{j}}{\partial x_{i}}=\frac{\partial K_{i}}{\partial p_{j}}, \quad \frac{\partial p_{j}}{\partial x_{i}}=-\frac{\partial K_{i}}{\partial q_{j}} \quad(i, j=1, \ldots, n)
$$

with polynomial Hamiltonians $K_{i}(i=1, \ldots, n)$. These $K_{i}$ are given explicitly by

$$
\begin{aligned}
x_{i}\left(x_{i}-1\right) K_{i}= & q_{i}\left(\rho+\sum_{j=1}^{n} q_{j} p_{j}\right)\left(\rho+\theta_{n+3}+1+\sum_{j=1}^{n} q_{j} p_{j}\right)+x_{i} p_{i}\left(q_{i} p_{i}-\theta_{i}\right) \\
& -\sum_{j=1, j \neq i}^{n} X_{i j} q_{i} p_{i}\left(q_{j} p_{j}-\theta_{j}\right)-\sum_{j=1, j \neq i}^{n} X_{j i} q_{i}\left(q_{j} p_{j}-\theta_{j}\right) p_{j} \\
& -\sum_{j=1, j \neq i}^{n} X_{i j}^{*}\left(q_{i} p_{i}-\theta_{i}\right) p_{i} q_{j}-\sum_{j=1, j \neq i}^{n} X_{i j}\left(q_{i} p_{i}-\theta_{i}\right) q_{j} p_{j} \\
& -\left(x_{i}+1\right)\left(q_{i} p_{i}-\theta_{i}\right) q_{i} p_{i}+\left(\theta_{n+2} x_{i}+\theta_{n+1}-1\right) q_{i} p_{i},
\end{aligned}
$$

where 


$$
X_{i j}=\frac{x_{i}\left(x_{j}-1\right)}{x_{j}-x_{i}}, \quad X_{i j}^{*}=\frac{x_{i}\left(x_{i}-1\right)}{x_{i}-x_{j}} .
$$

We call the Hamiltonian system (1.8) with the Hamiltonians (1.9) the Garnier system.

As is known in [1], the Garnier system is derived from the Schlesinger system (of rank 2). Then the independent and dependent variables of the Garnier system are expressed as certain rational functions in the variables of the Schlesinger system. Furthermore, the $\tau$-functions for the Garnier system can be identified with those for the Schlesinger system. Hence we first investigate symmetries and properties of the $\tau$-functions for the Schlesinger system. After that, we apply the obtained results to the Garnier system.

In Section 2, we give the transformations of three types, permutation of the points, sign change of the exponents and Schlesinger transformation, which act on the Schlesinger system. In Section 3, we formulate the $\tau$-functions for the Schlesinger system on the root lattice $Q\left(C_{n+3}\right)$. We also present bilinear relations which are satisfied by the $\tau$-functions. In Section 4 , we show that the Garnier system has affine Weyl group symmetry of type $B_{n+3}^{(1)}$.

\section{Schlesinger system}

Let $A_{j}$ and $G_{j}(j=1, \ldots, n+2)$ be matrices of dependent variables defined as

$$
A_{j}=\left(\begin{array}{cc}
a_{j} & b_{j} \\
c_{j} & d_{j}
\end{array}\right), \quad G_{j}=\left(\begin{array}{cc}
-d_{j} & b_{j} \\
c_{j} & d_{j}
\end{array}\right)\left(\begin{array}{cc}
g_{j} & 0 \\
0 & h_{j}
\end{array}\right) .
$$

Consider a system of total differential equations

$$
\begin{aligned}
& d A_{j}=\sum_{i=1, i \neq j}^{n+2}\left[A_{i}, A_{j}\right] d \log \left(t_{j}-t_{i}\right) \quad(j=1, \ldots, n+2), \\
& d G_{j}=\sum_{i=1, i \neq j}^{n+2} A_{i} G_{j} d \log \left(t_{j}-t_{i}\right) \quad(j=1, \ldots, n+2),
\end{aligned}
$$

where $t_{n+1}=0, t_{n+2}=1$ and $d$ is an exterior differentiation with respect to $t_{1}, \ldots, t_{n}$. Here we assume

(i) $\operatorname{det} A_{j}=0, \operatorname{tr} A_{j}=\theta_{j} \notin \boldsymbol{Z}(j=1, \ldots, n+2)$;

(ii) $-\sum_{j=1}^{n+2} A_{j}=\operatorname{diag}\left(\rho, \rho+\theta_{n+3}\right), \theta_{n+3} \notin \boldsymbol{Z}, \rho=-\sum_{j=1}^{n+3} \theta_{j} / 2$.

We call the system (2.2) the Schlesinger system.

Recall that the Schlesinger system is obtained as the compatibility condition for a system of linear differential equations on $\boldsymbol{P}^{1}(\boldsymbol{C})$ 


$$
\frac{\partial \vec{y}}{\partial z}=\sum_{j=1}^{n+2} \frac{A_{j}}{z-t_{j}} \vec{y}, \quad \frac{\partial \vec{y}}{\partial t_{i}}=-\frac{A_{i}}{z-t_{i}} \vec{y} \quad(i=1, \ldots, n),
$$

where $\vec{y}={ }^{t}\left(y_{1}, y_{2}\right)$ is a vector of unknown functions. The matrices $G_{j}$ $(j=1, \ldots, n+2)$ are obtained as follows. The system (2.3) has a local fundamental solution $Y=Y(z)$ of the form

$$
Y=Y_{j}\left(z-t_{j}\right)^{\theta_{j} E_{2}} \quad(j=1, \ldots, n+2),
$$

where

$$
E_{1}=\left(\begin{array}{ll}
1 & 0 \\
0 & 0
\end{array}\right), \quad E_{2}=\left(\begin{array}{ll}
0 & 0 \\
0 & 1
\end{array}\right) .
$$

Here $Y_{j}(z)$ is a $2 \times 2$ matrix which is holomorphic at $z=t_{j}$, such that

$$
\left.Y_{j}\right|_{z=t_{j}}=G_{j}, \quad G_{j}^{-1} A_{j} G_{j}=\theta_{j} E_{2} .
$$

Note that the Schlesinger system has an ambiguity for the following transformation:

$$
A_{j} \rightarrow C^{-1} A_{j} C, \quad G_{j} \rightarrow C^{-1} G_{j} \quad(j=1, \ldots, n+2),
$$

where

$$
C=\left(\begin{array}{cc}
\gamma_{1} & 0 \\
0 & \gamma_{2}
\end{array}\right) \quad\left(\gamma_{1}, \gamma_{2} \in C\right) .
$$

The Schlesinger system is invariant under the action of the following transformations of three types. They are associated with (1) permutation of the points $t_{1}, \ldots, t_{n+3}$, (2) sign change of the exponents $\theta_{1}, \ldots, \theta_{n+3}$, and (3) shifting of the exponents by integers (Schlesinger transformation). In this section, we describe these transformations.

\subsection{Permutation of the points}

In the following, we use the matrix notations

$$
w\left(A_{j}\right)=\left(\begin{array}{ll}
w\left(a_{j}\right) & w\left(b_{j}\right) \\
w\left(c_{j}\right) & w\left(d_{j}\right)
\end{array}\right)
$$

and

$$
w\left(G_{j}\right)=\left(\begin{array}{cc}
-w\left(d_{j}\right) & w\left(b_{j}\right) \\
w\left(c_{j}\right) & w\left(d_{j}\right)
\end{array}\right)\left(\begin{array}{cc}
w\left(g_{j}\right) & 0 \\
0 & w\left(h_{j}\right)
\end{array}\right)
$$

for a transformation $w$ of the dependent variables. 
The action of the symmetric group $\mathfrak{S}_{n+3}$ on the set of the points $t_{1}, \ldots, t_{n}$, $t_{n+1}=0, t_{n+2}=1, t_{n+3}=\infty$ can be lifted to transformations of the independent and dependent variables. Denoting the adjacent transpositions by $\sigma_{1}=$ $(12), \ldots, \sigma_{n+2}=(n+2, n+3)$, we describe the action of these $\sigma_{k}$ on the variables $t_{i}(i=1, \ldots, n)$ and $a_{j}, b_{j}, c_{j}, d_{j}, g_{j}, h_{j}(j=1, \ldots, n+2)$.

$$
\sigma_{k}\left(t_{i}\right)=t_{\sigma_{k}(i)}, \quad \sigma_{k}\left(A_{j}\right)=A_{\sigma_{k}(j)}, \quad \sigma_{k}\left(G_{j}\right)=G_{\sigma_{k}(j)}
$$

for $k=1, \ldots, n-1$. We remark that $\sigma_{n}, \sigma_{n+1}$ and $\sigma_{n+2}$ are derived from Mëbius transformations on $\boldsymbol{P}^{1}(\boldsymbol{C})$. The transformation $\sigma_{n}$ is derived from $z \rightarrow\left(z-t_{n}\right) /\left(1-t_{n}\right)$ :

$$
\begin{aligned}
& \sigma_{n}\left(t_{i}\right)=\frac{t_{i}-t_{n}}{1-t_{n}} \quad(i \neq n), \quad \sigma_{n}\left(t_{n}\right)=\frac{-t_{n}}{1-t_{n}}, \\
& \sigma_{n}\left(A_{j}\right)=\left(1-t_{n}\right)^{\theta_{n+3} E_{2}} A_{\sigma_{n}(j)}\left(1-t_{n}\right)^{-\theta_{n+3} E_{2}}, \\
& \sigma_{n}\left(G_{j}\right)=\left(1-t_{n}\right)^{\rho I_{2}+\theta_{n+3} E_{2}} G_{\sigma_{n}(j)}\left(1-t_{n}\right)^{\theta_{\sigma_{n}(j)} E_{2}} .
\end{aligned}
$$

Similarly, the transformation $\sigma_{n+1}$ is derived from $z \rightarrow 1-z$ :

$$
\sigma_{n+1}\left(t_{i}\right)=1-t_{i}, \quad \sigma_{n+1}\left(A_{j}\right)=A_{\sigma_{n+1}(j)}, \quad \sigma_{n+1}\left(G_{j}\right)=G_{\sigma_{n+1}(j)},
$$

and the transformation $\sigma_{n+2}$ is derived from $z \rightarrow 1 / z$ :

$$
\begin{array}{ll}
\sigma_{n+2}\left(t_{i}\right)=\frac{t_{i}}{t_{i}-1}, & \\
\sigma_{n+2}\left(A_{j}\right)=G_{n+2}^{-1} A_{j} G_{n+2} & (j \neq n+2), \\
\sigma_{n+2}\left(A_{n+2}\right)=\theta_{n+3} G_{n+2}^{-1} E_{2} G_{n+2}, & \\
\sigma_{n+2}\left(G_{j}\right)=G_{n+2}^{-1} G_{j}\left(t_{j}-1\right)^{\rho I_{2}+2 \theta_{j} E_{2}} & (j \neq n+2), \\
\sigma_{n+2}\left(G_{n+2}\right)=G_{n+2}^{-1}, &
\end{array}
$$

The action of each $\sigma_{k}$ on the parameters $\theta_{j}$ is given by

$$
\sigma_{k}\left(\theta_{j}\right)=\theta_{\sigma_{k}(j)} \quad(j=1, \ldots, n+3) .
$$

\subsection{Sign change of the exponents}

Let $Y$ be a fundamental solution of system (2.3). Consider the gauge transformations

$$
r_{k}(Y)=\left(z-t_{k}\right)^{-\theta_{k}} Y \quad(k=1, \ldots, n+2), \quad r_{n+3}(Y)=W Y,
$$

where 


$$
W=\left(\begin{array}{ll}
0 & 1 \\
1 & 0
\end{array}\right) .
$$

Each $r_{k}$ acts on the parameters as follows:

$$
r_{k}\left(\theta_{j}\right)=(-1)^{\delta_{j k}} \theta_{j} \quad(j=1, \ldots, n+3),
$$

where $\delta_{j k}$ stands for the Kronecker delta, and can be lifted to a transformation of the dependent variables. We describe the action of these $r_{k}$.

$$
\begin{array}{ll}
r_{k}\left(A_{j}\right)=A_{j}-\delta_{j k} \theta_{k} I_{2} & (j=1, \ldots, n+2), \\
r_{k}\left(G_{j}\right)=\left(t_{j}-t_{k}\right)^{-\delta_{j k} \theta_{k}} G_{j} & (j=1, \ldots, n+2)
\end{array}
$$

for $k=1, \ldots, n+2$.

$$
r_{n+3}\left(A_{j}\right)=W A_{j} W, \quad r_{n+3}\left(G_{j}\right)=W G_{j} \quad(j=1, \ldots, n+2)
$$

for $k=n+3$. Note that the independent variables $t_{i}(i=1, \ldots, n)$ are invariant under the action of each $r_{k}$.

\subsection{Schlesinger transformations}

In this section, we construct the Schlesinger transformations by following [3]. Let $L$ be a subset of $\boldsymbol{Z}^{n+3}$ defined as

$$
L=\left\{\mu=\left(\mu_{1}, \ldots, \mu_{n+3}\right) \in \boldsymbol{Z}^{n+3} \mid \mu_{1}+\cdots+\mu_{n+3} \in 2 \boldsymbol{Z}\right\} .
$$

Consider the gauge transformations

$$
T_{\mu}(Y)=R_{\mu} Y \quad(\mu \in L),
$$

where $R_{\mu}$ are $2 \times 2$ matrices of rational functions in $z$ and $t_{i}(i=1, \ldots, n)$, such that

$$
T_{\mu}\left(\theta_{j}\right)=\theta_{j}+\mu_{j} \quad(j=1, \ldots, n+3) .
$$

Then each $R_{\mu}$ is determined up to multiplication by a scalar matrix and the gauge transformation $T_{\mu}$ can be lifted to a birational transformation (called the Schlesinger transformation) of the dependent variables.

The group of the Schlesinger transformations is generated by the transformations $T_{k}(k=1, \ldots, n+2)$, such that

$$
T_{k}\left(\theta_{j}\right)=\theta_{j}+\delta_{j k}-\delta_{j k+1} \quad(j=1, \ldots, n+3),
$$

and $T_{n+3}$, such that

$$
T_{n+3}\left(\theta_{j}\right)=\theta_{j}+\delta_{j n+2}+\delta_{j n+3} \quad(j=1, \ldots, n+3) .
$$


We describe the action of these $T_{k}$ on the variables $a_{j}, b_{j}, c_{j}, d_{j}, g_{j}, h_{j}$ $(j=1, \ldots, n+2)$.

$$
\begin{aligned}
T_{k}\left(A_{j}\right) & =A_{j}+\frac{R_{k}^{*} A_{j} R_{k}}{\left(t_{k}-t_{k+1}\right)\left(t_{k}-t_{j}\right)}-\frac{R_{k} A_{j} R_{k}^{*}}{\left(t_{k}-t_{k+1}\right)\left(t_{k+1}-t_{j}\right)} \quad(j \neq k, k+1), \\
T_{k}\left(A_{k}\right) & =A_{k+1}-\frac{\left(1+\theta_{k}-\theta_{k+1}\right) R_{k}}{t_{k}-t_{k+1}}-\sum_{j=1, j \neq k, k+1}^{n+2} \frac{R_{k}^{*} A_{j} R_{k}}{\left(t_{k}-t_{k+1}\right)\left(t_{k}-t_{j}\right)}, \\
T_{k}\left(A_{k+1}\right) & =A_{k}+\frac{\left(1+\theta_{k}-\theta_{k+1}\right) R_{k}}{t_{k}-t_{k+1}}+\sum_{j=1, j \neq k, k+1}^{n+2} \frac{R_{k} A_{j} R_{k}^{*}}{\left(t_{k}-t_{k+1}\right)\left(t_{k+1}-t_{j}\right)}, \\
T_{k}\left(G_{j}\right) & =G_{j}-\frac{R_{k} G_{j}}{t_{k+1}-t_{j}}(j \neq k, k+1), \\
T_{k}\left(G_{k}\right) & =\frac{R_{k}^{*} G_{k}}{t_{k}-t_{k+1}}+\frac{G_{k} E_{2}}{t_{k}-t_{k+1}}+\sum_{j=1, j \neq k}^{n+2} \frac{R_{k}^{*} G_{k} E_{1} G_{k}^{-1} A_{j} G_{k} E_{2}}{\left(1+\theta_{k}\right)\left(t_{k}-t_{k+1}\right)\left(t_{k}-t_{j}\right)}, \\
T_{k}\left(G_{k+1}\right) & =R_{k} G_{k+1}-G_{k+1} E_{2}+\sum_{j=1, j \neq k}^{n+2} \frac{R_{k} G_{k+1} E_{2} G_{k}^{-1} A_{j} G_{k} E_{1}}{\left(1-\theta_{k}\right)\left(t_{k}-t_{j}\right)},
\end{aligned}
$$

where

$$
R_{k}=\frac{-t_{k}+t_{k+1}}{b_{k} a_{k+1}+d_{k} b_{k+1}}\left(\begin{array}{c}
b_{k} \\
d_{k}
\end{array}\right)\left(\begin{array}{ll}
a_{k+1} & b_{k+1}
\end{array}\right), \quad R_{k}^{*}=\left(t_{k}-t_{k+1}\right) I_{2}+R_{k}
$$

for $k=1, \ldots, n+1$.

$$
\begin{gathered}
T_{n+2}\left(A_{n+2}\right)=R_{n+2} A_{n+2} E_{1}+E_{2} A_{n+2} R_{n+2}^{*}+E_{2} R_{n+2}^{*}-\sum_{j=1}^{n+1} \frac{R_{n+2} A_{j} R_{n+2}^{*}}{t_{j}-1}, \\
T_{n+2}\left(A_{j}\right)=\left(t_{j}-1\right) E_{2} A_{j} E_{1}+R_{n+2} A_{j} E_{1}+E_{2} A_{j} R_{n+2}^{*}+\frac{R_{n+2} A_{j} R_{n+2}^{*}}{t_{j}-1} \\
\quad(j \neq n+2), \\
T_{n+2}\left(G_{n+2}\right)=R_{n+2} G_{n+2}+E_{2} G_{n+2} E_{2}+\sum_{j=1, j \neq k}^{n+2} \frac{R_{n+2} G_{n+2} E_{1} G_{n+2}^{-1} A_{j} G_{n+2} E_{2}}{\left(1+\theta_{n+2}\right)\left(t_{n+2}-t_{j}\right)}, \\
T_{n+2}\left(G_{j}\right)=\left(t_{j}-1\right) E_{2} G_{j}+R_{n+2} G_{j} \quad(j \neq n+2),
\end{gathered}
$$

where 


$$
\begin{aligned}
& R_{n+2}=\frac{1}{\left(1-\theta_{n+3}\right) d_{n+2}}\left(\begin{array}{c}
1-\theta_{n+3} \\
c_{\infty}
\end{array}\right)\left(d_{n+2}-b_{n+2}\right), \\
& R_{n+2}^{*}=\frac{1}{\left(1-\theta_{n+3}\right) d_{n+2}}\left(\begin{array}{c}
b_{n+2} \\
d_{n+2}
\end{array}\right)\left(\begin{array}{ll}
-c_{\infty} & 1-\theta_{n+3}
\end{array}\right)
\end{aligned}
$$

and $c_{\infty}=\sum_{j=1}^{n+2} t_{j} c_{j}$, for $k=n+2$.

$$
\begin{gathered}
T_{n+3}\left(A_{n+2}\right)=R_{n+3} A_{n+2} E_{2}+E_{1} A_{n+2} R_{n+3}^{*}+E_{1} R_{n+3}^{*}-\sum_{j=1}^{n+1} \frac{R_{n+3} A_{j} R_{n+3}^{*}}{t_{j}-1}, \\
T_{n+3}\left(A_{j}\right)=\left(t_{j}-1\right) E_{1} A_{j} E_{2}+R_{n+3} A_{j} E_{2}+E_{1} A_{j} R_{n+3}^{*}+\frac{R_{n+3} A_{j} R_{n+3}^{*}}{t_{j}-1} \\
(j \neq n+2), \\
T_{n+3}\left(G_{n+2}\right)=R_{n+3} G_{n+2}+E_{1} G_{n+2} E_{2}+\sum_{j=1, j \neq k}^{n+2} \frac{R_{n+3} G_{n+2} E_{1} G_{n+2}^{-1} A_{j} G_{n+2} E_{2}}{\left(1+\theta_{n+2}\right)\left(t_{n+2}-t_{j}\right)}, \\
T_{n+3}\left(G_{j}\right)=\left(t_{j}-1\right) E_{1} G_{j}+R_{n+3} G_{j} \quad(j \neq n+2),
\end{gathered}
$$

where

$$
\begin{aligned}
& R_{n+3}=\frac{1}{\left(1+\theta_{n+3}\right) b_{n+2}}\left(\begin{array}{c}
b_{\infty} \\
1+\theta_{n+3}
\end{array}\right)\left(\begin{array}{ll}
-d_{n+2} & b_{n+2}
\end{array}\right), \\
& R_{n+3}^{*}=\frac{1}{\left(1+\theta_{n+3}\right) b_{n+2}}\left(\begin{array}{l}
b_{n+2} \\
d_{n+2}
\end{array}\right)\left(1+\theta_{n+3}-b_{\infty}\right)
\end{aligned}
$$

and $b_{\infty}=\sum_{j=1}^{n+2} t_{j} b_{j}$, for $k=n+3$. Note that the independent variables $t_{i}$ $(i=1, \ldots, n)$ are invariant under the action of each $T_{k}$.

Remark 2.1. The group of the Schlesinger transformations generated by $T_{k}(k=1, \ldots, n+3)$ is isomorphic to the root lattice $Q\left(C_{n+3}\right)$. The commutativity between two arbitrary Schlesinger transformations is obtained from the uniqueness of the Schlesinger transformations [3].

\section{3. $\tau$-Functions on the root lattice}

In this Section, we formulate the $\tau$-functions for the Schlesinger system on the root lattice $Q\left(C_{n+3}\right)$. We also present the bilinear relations of three types, Toda equations, Hirota-Miwa equations and bilinear differential equations, which are satisfied by the $\tau$-functions.

Proposition 3.1 ([3]). For each solution of the Schlesinger system, the 1-forms 


$$
\omega_{\mu}=\sum_{i=1}^{n} T_{\mu}\left(H_{i}\right) d t_{i} \quad(\mu \in L)
$$

are closed. Here we let

$$
H_{i}=\sum_{j=1, j \neq i}^{n+2} \frac{1}{t_{i}-t_{j}}\left(\operatorname{tr} A_{i} A_{j}+C_{i j}\right) \quad(i=1, \ldots, n),
$$

where

$$
C_{i j}=-\frac{1}{2} \theta_{i} \theta_{j}+\frac{\theta_{i}^{2}+\theta_{j}^{2}}{2(n+1)}-\frac{\sum_{i=1}^{n+3} \theta_{i}^{2}}{2(n+1)(n+2)} .
$$

Proposition 3.1 allows us to define a family of $\tau$-functions by

$$
d \log \tau_{\mu}=\omega_{\mu} \quad(\mu \in L),
$$

up to multiplicative constants.

We also define the action of the transformations $\sigma_{k}, r_{l}$ and $T_{\mu}$ on the $\tau$-functions, so that it is consistent with the action of them on $H_{i}$ which we call Hamiltonians. For each $\mu, v \in L$, the action of $T_{\mu}$ on $\tau_{v}$ is defined by

$$
T_{\mu}\left(\tau_{v}\right)=\tau_{\mu+v}
$$

and the action of $\sigma_{k}, r_{l}$ on $\tau_{v}$ is defined by

$$
\begin{array}{ll}
\sigma_{k}\left(\tau_{v}\right)=\tau_{\sigma_{k}(v)} & (k=1, \ldots, n+2), \\
r_{l}\left(\tau_{v}\right)=\tau_{r_{l}(v)} & (l=1, \ldots, n+3),
\end{array}
$$

where

$$
\begin{aligned}
\sigma_{k}(v) & =\left(v_{\sigma_{k}(1)}, \ldots, v_{\sigma_{k}(n+3)}\right) \\
r_{l}(v) & =\left(v_{1}, \ldots, v_{l-1},-v_{l}, v_{l+1}, \ldots, v_{n+3}\right) .
\end{aligned}
$$

In Section 3.1, we describe the action of the transformations $\sigma_{k}, r_{l}$ and $T_{\mu}$ on the Hamiltonians, which is obtained from the action of them on the independent and dependent variables.

\subsection{Symmetries for Hamiltonians}

We first describe the action of the Schlesinger transformation $T_{\mu}$ on the Hamiltonians for each $\mu \in L$ with

$$
\mu_{1}^{2}+\cdots+\mu_{n+3}^{2}=2 .
$$

Set 


$$
\begin{gathered}
T_{k, l}=T_{\mathbf{e}_{k}+\mathbf{e}_{l}}, \quad T_{k,-l}=T_{\mathbf{e}_{k}-\mathbf{e}_{l}}, \quad T_{-k,-l}=T_{-\mathbf{e}_{k}-\mathbf{e}_{l}} \\
(k, l=1, \ldots, n+3, k \neq l),
\end{gathered}
$$

where

$$
\begin{aligned}
\mathbf{e}_{1} & =(1,0,0, \ldots, 0,0), \\
\mathbf{e}_{2} & =(0,1,0, \ldots, 0,0), \\
& \vdots \\
\mathbf{e}_{n+3} & =(0,0,0, \ldots, 0,1) .
\end{aligned}
$$

We remark

$$
T_{k}=T_{k,-(k+1)} \quad(k=1, \ldots, n+2), \quad T_{n+3}=T_{n+2, n+3}
$$

and that they act on $\theta_{j}(j=1, \ldots, n+3)$ as follows:

$$
\begin{array}{r}
T_{k, l}\left(\theta_{j}\right)=\theta_{j}+\delta_{j k}+\delta_{j l}, \\
T_{k,-l}\left(\theta_{j}\right)=\theta_{j}+\delta_{j k}-\delta_{j l}, \\
T_{-k,-l}\left(\theta_{j}\right)=\theta_{j}-\delta_{j k}-\delta_{j l} .
\end{array}
$$

Then the action of them on the Hamiltonians $H_{i}(i=1, \ldots, n)$ is described as follows.

$$
\begin{aligned}
T_{k, l}\left(H_{i}\right)= & H_{i}-\frac{\operatorname{tr} A_{i} R_{k, l}}{\left(t_{i}-t_{k}\right)\left(t_{i}-t_{l}\right)}+\frac{\Gamma_{k}^{i}}{t_{i}-t_{k}}+\frac{\Gamma_{l}^{-i}}{t_{i}-t_{l}}+\sum_{j=1, j \neq i}^{n+2} \frac{\Gamma_{k, l}}{t_{i}-t_{j}} \quad(i \neq k, l), \\
T_{k, l}\left(H_{k}\right)= & H_{k}-\sum_{j=1, j \neq k, l}^{n+2} \frac{\operatorname{tr} A_{j} R_{k, l}}{\left(t_{k}-t_{j}\right)\left(t_{k}-t_{l}\right)}-\frac{(n-1)\left(1+\theta_{k}+\theta_{l}\right)}{2(n+1)\left(t_{k}-t_{l}\right)} \\
& +\sum_{j=1, j \neq k, l}^{n+2} \frac{\Gamma_{k}^{j}}{t_{k}-t_{j}}+\sum_{j=1, j \neq k}^{n+2} \frac{\Gamma_{k, l}}{t_{k}-t_{j}}, \\
T_{k, l}\left(H_{l}\right)= & H_{l}-\sum_{j=1, j \neq k, l}^{n+2} \frac{\operatorname{tr} A_{j} R_{k, l}}{\left(t_{l}-t_{j}\right)\left(t_{l}-t_{k}\right)}-\frac{(n-1)\left(1+\theta_{k}+\theta_{l}\right)}{2(n+1)\left(t_{l}-t_{k}\right)} \\
& +\sum_{j=1, j \neq k, l}^{n+2} \frac{\Gamma_{k}^{-j}}{t_{l}-t_{j}}+\sum_{j=1, j \neq l}^{n+2} \frac{\Gamma_{k, l}}{t_{l}-t_{j}},
\end{aligned}
$$

where 


$$
\begin{array}{ll}
\Gamma_{k}^{j}=-\frac{\theta_{j}}{2}+\frac{1+2 \theta_{k}}{2(n+2)}, & \Gamma_{k}^{-j}=\frac{\theta_{j}}{2}+\frac{1+2 \theta_{k}}{2(n+2)}, \\
\Gamma_{k, l}=-\frac{1+\theta_{k}+\theta_{l}}{(n+1)(n+2)}, & R_{k, l}=\frac{t_{k}-t_{l}}{b_{k} d_{l}-d_{k} b_{l}}\left(\begin{array}{c}
b_{k} \\
d_{k}
\end{array}\right)\left(\begin{array}{ll}
-d_{l} & b_{l}
\end{array}\right),
\end{array}
$$

for $k, l=1, \ldots, n+2$ with $k \neq l$.

$$
\begin{aligned}
T_{k,-l}\left(H_{i}\right)= & H_{i}-\frac{\operatorname{tr} A_{i} R_{k,-l}}{\left(t_{i}-t_{k}\right)\left(t_{i}-t_{l}\right)}+\frac{\Gamma_{k}^{i}}{t_{i}-t_{k}}+\frac{\Gamma_{-l}^{-i}}{t_{i}-t_{l}}+\sum_{j=1, j \neq i}^{n+2} \frac{\Gamma_{k,-l}}{t_{i}-t_{j}} \quad(i \neq k, l), \\
T_{k,-l}\left(H_{k}\right)= & H_{k}-\sum_{j=1, j \neq k, l}^{n+2} \frac{\operatorname{tr} A_{j} R_{k,-l}}{\left(t_{k}-t_{j}\right)\left(t_{k}-t_{l}\right)}-\frac{(n-1)\left(1+\theta_{k}-\theta_{l}\right)}{2(n+1)\left(t_{k}-t_{l}\right)} \\
& +\sum_{j=1, j \neq k, l}^{n+2} \frac{\Gamma_{k}^{j}}{t_{k}-t_{j}}+\sum_{j=1, j \neq k}^{n+2} \frac{\Gamma_{k,-l}}{t_{k}-t_{j}}, \\
T_{k,-l}\left(H_{l}\right)= & H_{l}-\sum_{j=1, j \neq k, l}^{n+2} \frac{\operatorname{tr} A_{j} R_{k,-l}}{\left(t_{l}-t_{j}\right)\left(t_{l}-t_{k}\right)}-\frac{(n-1)\left(1+\theta_{k}-\theta_{l}\right)}{2(n+1)\left(t_{l}-t_{k}\right)} \\
& +\sum_{j=1, j \neq k, l}^{n+2} \frac{\Gamma_{-l}^{-j}}{t_{l}-t_{j}}+\sum_{j=1, j \neq l}^{n+2} \frac{\Gamma_{k,-l}}{t_{l}-t_{j}},
\end{aligned}
$$

where

$$
\begin{aligned}
\Gamma_{k,-l} & =-\frac{1+\theta_{k}-\theta_{l}}{(n+1)(n+2)}, \quad \Gamma_{-k}^{-j}=\frac{\theta_{j}}{2}+\frac{1-2 \theta_{k}}{2(n+2)}, \\
R_{k,-l} & =\frac{-t_{k}+t_{l}}{b_{k} a_{l}+d_{k} b_{l}}\left(\begin{array}{c}
b_{k} \\
d_{k}
\end{array}\right)\left(\begin{array}{ll}
a_{l} & \left.b_{l}\right),
\end{array}\right.
\end{aligned}
$$

for $k, l=1, \ldots, n+2$ with $k \neq l$.

$$
\begin{aligned}
& T_{-k,-l}\left(H_{i}\right)= H_{i}-\frac{\operatorname{tr} A_{i} R_{-k,-l}}{\left(t_{i}-t_{k}\right)\left(t_{i}-t_{l}\right)}+\frac{\Gamma_{-k}^{i}}{t_{i}-t_{k}}+\frac{\Gamma_{-l}^{-i}}{t_{i}-t_{l}}+\sum_{j=1, j \neq i}^{n+2} \frac{\Gamma_{-k,-l}}{t_{i}-t_{j}} \\
& \quad(i \neq k, l), \\
& T_{-k,-l}\left(H_{k}\right)= H_{k}-\sum_{j=1, j \neq k, l}^{n+2} \frac{\operatorname{tr} A_{j} R_{-k,-l}}{\left(t_{k}-t_{j}\right)\left(t_{k}-t_{l}\right)}-\frac{(n-1)\left(1-\theta_{k}-\theta_{l}\right)}{2(n+1)\left(t_{k}-t_{l}\right)} \\
&+\sum_{j=1, j \neq k, l}^{n+2} \frac{\Gamma_{-k}^{j}}{t_{k}-t_{j}}+\sum_{j=1, j \neq k}^{n+2} \frac{\Gamma_{-k,-l}}{t_{k}-t_{j}},
\end{aligned}
$$




$$
\begin{aligned}
T_{-k,-l}\left(H_{l}\right)= & H_{l}-\sum_{j=1, j \neq k, l}^{n+2} \frac{\operatorname{tr} A_{j} R_{-k,-l}}{\left(t_{l}-t_{j}\right)\left(t_{l}-t_{k}\right)}-\frac{(n-1)\left(1-\theta_{k}-\theta_{l}\right)}{2(n+1)\left(t_{l}-t_{k}\right)} \\
& +\sum_{j=1, j \neq k, l}^{n+2} \frac{\Gamma_{-l}^{-j}}{t_{l}-t_{j}}+\sum_{j=1, j \neq l}^{n+2} \frac{\Gamma_{-k,-l}}{t_{l}-t_{j}}
\end{aligned}
$$

where

$$
\begin{aligned}
\Gamma_{-k,-l} & =-\frac{1-\theta_{k}-\theta_{l}}{(n+1)(n+2)}, \quad \Gamma_{-k}^{j}=-\frac{\theta_{j}}{2}+\frac{1-2 \theta_{k}}{2(n+2)}, \\
R_{-k,-l} & =\frac{t_{k}-t_{l}}{a_{k} b_{l}-b_{k} a_{l}}\left(\begin{array}{c}
b_{k} \\
-a_{k}
\end{array}\right)\left(\begin{array}{ll}
a_{l} & b_{l}
\end{array}\right),
\end{aligned}
$$

for $k, l=1, \ldots, n+2$ with $k \neq l$.

$$
\begin{aligned}
& T_{k, n+3}\left(H_{i}\right)=H_{i}+\frac{1}{t_{i}-t_{k}}\left(a_{i}+b_{i} \frac{d_{k}}{b_{k}}\right)+\frac{\Gamma_{k}^{i}}{t_{i}-t_{k}}+\sum_{j=1, j \neq i}^{n+2} \frac{\Gamma_{k, n+3}}{t_{i}-t_{j}} \quad(i \neq k), \\
& T_{k, n+3}\left(H_{k}\right)=H_{k}+\sum_{j=1, j \neq k}^{n+2} \frac{1}{t_{k}-t_{j}}\left(a_{j}+b_{j} \frac{d_{k}}{b_{k}}\right)+\sum_{j=1, j \neq k}^{n+2} \frac{\Gamma_{k}^{j}+\Gamma_{k, n+3}}{t_{k}-t_{j}}
\end{aligned}
$$

for $k=1, \ldots, n+2$.

$$
\begin{gathered}
T_{k,-(n+3)}\left(H_{i}\right)=H_{i}+\frac{1}{t_{i}-t_{k}}\left(d_{i}+c_{i} \frac{a_{k}}{c_{k}}\right)+\frac{\Gamma_{k}^{i}}{t_{i}-t_{k}}+\sum_{j=1, j \neq i}^{n+2} \frac{\Gamma_{k,-(n+3)}}{t_{i}-t_{j}} \\
(i \neq k), \\
T_{k,-(n+3)}\left(H_{k}\right)=H_{k}+\sum_{j=1, j \neq k}^{n+2} \frac{1}{t_{k}-t_{j}}\left(d_{j}+c_{j} \frac{a_{k}}{c_{k}}\right)+\sum_{j=1, j \neq k}^{n+2} \frac{\Gamma_{k}^{j}+\Gamma_{k,-(n+3)}}{t_{k}-t_{j}}
\end{gathered}
$$

for $k=1, \ldots, n+2$.

$$
\begin{aligned}
& T_{n+3,-k}\left(H_{i}\right)=H_{i}+\frac{1}{t_{i}-t_{k}}\left(a_{i}-b_{i} \frac{a_{k}}{b_{k}}\right)+\frac{\Gamma_{-k}^{i}}{t_{i}-t_{k}}+\sum_{j=1, j \neq i}^{n+2} \frac{\Gamma_{n+3,-k}}{t_{i}-t_{j}} \quad(i \neq k), \\
& T_{n+3,-k}\left(H_{k}\right)=H_{k}+\sum_{j=1, j \neq k}^{n+2} \frac{1}{t_{k}-t_{j}}\left(a_{j}-b_{j} \frac{a_{k}}{b_{k}}\right)+\sum_{j=1, j \neq k}^{n+2} \frac{\Gamma_{-k}^{j}+\Gamma_{n+3,-k}}{t_{k}-t_{j}}
\end{aligned}
$$

for $k=1, \ldots, n+2$. 


$$
\begin{aligned}
& T_{-k,-(n+3)}\left(H_{i}\right)=H_{i}+\frac{1}{t_{i}-t_{k}}\left(d_{i}-c_{i} \frac{d_{k}}{c_{k}}\right)+\frac{\Gamma_{-k}^{i}}{t_{i}-t_{k}}+\sum_{j=1, j \neq i}^{n+2} \frac{\Gamma_{-k,-(n+3)}}{t_{i}-t_{j}} \quad(i \neq k), \\
& T_{-k,-(n+3)}\left(H_{k}\right)=H_{k}+\sum_{j=1, j \neq k}^{n+2} \frac{1}{t_{k}-t_{j}}\left(d_{j}-c_{j} \frac{d_{k}}{c_{k}}\right)+\sum_{j=1, j \neq k}^{n+2} \frac{\Gamma_{-k}^{j}+\Gamma_{-k,-(n+3)}}{t_{k}-t_{j}}
\end{aligned}
$$

for $k=1, \ldots, n+2$. For the other $\mu \in L$, the action of $T_{\mu}$ on the Hamiltonians, which is not described in this paper, is similarly obtained from its action on the dependent variables.

Next we describe the action of the transformations $\sigma_{k}(k=1, \ldots, n+2)$ and $r_{l}(l=1, \ldots, n+3)$ on the Hamiltonians. Since $H_{i}(i=1, \ldots, n)$ are invariant under the action of each $\sigma_{k}$ and $r_{l}$, we obtain

$$
\sigma_{k} T_{\mu}\left(H_{i}\right)=T_{\sigma_{k}(\mu)}\left(H_{i}\right), \quad r_{l} T_{\mu}\left(H_{i}\right)=T_{r_{l}(\mu)}\left(H_{i}\right) \quad(\mu \in L),
$$

where

$$
\begin{aligned}
\sigma_{k}(\mu) & =\left(\mu_{\sigma_{k}(1)}, \ldots, \mu_{\sigma_{k}(n+3)}\right) \\
r_{l}(\mu) & =\left(\mu_{1}, \ldots, \mu_{l-1},-\mu_{l}, \mu_{l+1}, \ldots, \mu_{n+3}\right) .
\end{aligned}
$$

\subsection{Toda equations}

In this section, we present the Toda equations for the Schlesinger transformations $T_{k}(k=1, \ldots, n+3)$. Set

$$
\tilde{H}_{i}=H_{i}-\sum_{j=1, j \neq i}^{n+2} \frac{C_{i j}}{t_{i}-t_{j}}=\sum_{j=1, j \neq i}^{n+2} \frac{\operatorname{tr} A_{i} A_{j}}{t_{i}-t_{j}} \quad(i=1, \ldots, n) .
$$

Then we have

Theorem $3.2([3])$. The Hamiltonians $\tilde{H}_{i}(i=1, \ldots, n)$ satisfy the following equations:

$$
\begin{gathered}
T_{k}\left(\tilde{H}_{i}\right)+T_{k}^{-1}\left(\tilde{H}_{i}\right)-2 \tilde{H}_{i}=\partial_{t_{i}} \log \frac{\left(G_{k+1}^{-1} G_{k}\right)_{22}\left(G_{k}^{-1} G_{k+1}\right)_{22}}{\left(t_{k}-t_{k+1}\right)^{2}} \\
(k=1, \ldots, n+1), \\
T_{n+2}\left(\tilde{H}_{i}\right)+T_{n+2}^{-1}\left(\tilde{H}_{i}\right)-2 \tilde{H}_{i}=\partial_{t_{i}} \log \left(G_{n+2}\right)_{22}\left(G_{n+2}^{-1}\right)_{22}, \\
T_{n+3}\left(\tilde{H}_{i}\right)+T_{n+3}^{-1}\left(\tilde{H}_{i}\right)-2 \tilde{H}_{i}=\partial_{t_{i}} \log \left(G_{n+2}\right)_{12}\left(G_{n+2}^{-1}\right)_{21},
\end{gathered}
$$

where $\left(G_{j}\right)_{k l}$ stands for the $(k, l)$-component of the $2 \times 2$ matrix $G_{j}$. 
We also obtain the following lemma.

Lemma 3.3. The Hamiltonians $\tilde{H}_{i}(i=1, \ldots, n)$ satisfy the following equations:

$$
\begin{aligned}
\partial_{t_{k}}\left(\tilde{H}_{k+1}\right) & =\frac{\operatorname{tr} A_{k} A_{k+1}}{\left(t_{k}-t_{k+1}\right)^{2}} \quad(k=1, \ldots, n-1), \\
\partial_{t_{n}}\left(\sum_{i=1}^{n}\left(t_{i}-1\right) \tilde{H}_{i}\right) & =\frac{\operatorname{tr} A_{n} A_{n+1}}{t_{n}^{2}}, \\
\left(\delta^{*}+1\right)\left(\sum_{i=1}^{n} t_{i} \tilde{H}_{i}\right) & =-\operatorname{tr} A_{n+1} A_{n+2}-\frac{1}{2} \sum_{i=1}^{n+2} \sum_{j=1, j \neq i}^{n+2} C_{i j}, \\
(\delta+1)\left(\sum_{i=1}^{n} t_{i} \tilde{H}_{i}\right) & =\theta_{n+3} d_{n+2}+\theta_{n+2}\left(\rho+\theta_{n+2}\right)-\frac{1}{2} \sum_{i=1}^{n+2} \sum_{j=1, j \neq i}^{n+2} C_{i j},
\end{aligned}
$$

where $\partial_{i}=\partial / \partial t_{i}$ and

$$
\delta=\sum_{i=1}^{n} t_{i}\left(t_{i}-1\right) \partial_{t_{i}}, \quad \delta^{*}=\sum_{i=1}^{n}\left(t_{i}-1\right) \partial_{t_{i}}
$$

Proof. The first equation of (3.27) is obtained by a direct computation. The second equation of (3.27) is obtained by using

$$
\sum_{i=1}^{n}\left(t_{i}-1\right) \tilde{H}_{i}=-\sum_{j=1, j \neq n+1}^{n+2} \frac{\operatorname{tr} A_{j} A_{n+1}}{t_{j}}+\sum_{i=1}^{n+2} \sum_{j=1, j \neq i}^{n+2} \operatorname{tr} A_{i} A_{j}
$$

and

$$
\sum_{i=1}^{n+2} \sum_{j=1, j \neq i}^{n+2} \operatorname{tr} A_{i} A_{j}=-\sum_{i=1}^{n+2} \sum_{j=1, j \neq i}^{n+2} C_{i j}
$$

The third equation of (3.27) is obtained by using (3.30),

$$
\sum_{i=1}^{n} t_{i} \tilde{H}_{i}=\sum_{j=1}^{n+1} \frac{\operatorname{tr} A_{j} A_{n+2}}{t_{j}-1}+\frac{1}{2} \sum_{i=1}^{n+2} \sum_{j=1, j \neq i}^{n+2} \operatorname{tr} A_{i} A_{j}
$$

and 


$$
\left(\delta^{*}+1\right)\left(\sum_{j=1}^{n+1} \frac{\operatorname{tr} A_{j} A_{n+2}}{t_{j}-1}\right)=-\operatorname{tr} A_{n+1} A_{n+2} .
$$

The fourth equation of (3.27) is obtained by using (3.30), (3.31) and

$$
(\delta+1)\left(\sum_{j=1}^{n+1} \frac{\operatorname{tr} A_{j} A_{n+2}}{t_{j}-1}\right)=\theta_{n+3} d_{n+2}+\theta_{n+2}\left(\rho+\theta_{n+2}\right) .
$$

From Theorem 3.2, Lemma 3.3 and the following identities:

$$
\begin{aligned}
\left(G_{k+1}^{-1} G_{k}\right)_{22}\left(G_{k}^{-1} G_{k+1}\right)_{22} & =-\frac{\operatorname{tr} A_{k} A_{k+1}}{\theta_{k} \theta_{k+1}} \quad(k=1, \ldots, n+1), \\
\left(G_{n+2}\right)_{22}\left(G_{n+2}^{-1}\right)_{22} & =\frac{d_{n+2}}{\theta_{n+2}} \\
\left(G_{n+2}\right)_{12}\left(G_{n+2}^{-1}\right)_{21} & =\frac{a_{n+2}}{\theta_{n+2}}
\end{aligned}
$$

we obtain

$$
T_{k}\left(\tilde{H}_{i}\right)+T_{k}^{-1}\left(\tilde{H}_{i}\right)-2 \tilde{H}_{i}=\partial_{t_{i}} \log X_{k} \quad(k=1, \ldots, n+3),
$$

where

$$
\begin{aligned}
X_{k} & =\partial_{t_{k}}\left(\tilde{H}_{k+1}\right) \quad(k=1, \ldots, n-1), \\
X_{n} & =\partial_{t_{n}}\left(\sum_{i=1}^{n}\left(t_{i}-1\right) \tilde{H}_{i}\right), \\
X_{n+1} & =\left(\delta^{*}+1\right)\left(\sum_{i=1}^{n} t_{i} \tilde{H}_{i}\right)+\frac{1}{2} \sum_{i=1}^{n+2} \sum_{j=1, j \neq i}^{n+2} C_{i j}, \\
X_{n+2} & =(\delta+1)\left(\sum_{i=1}^{n} t_{i} \tilde{H}_{i}\right)+\frac{1}{2} \sum_{i=1}^{n+2} \sum_{j=1, j \neq i}^{n+2} C_{i j}-\theta_{n+2}\left(\rho+\theta_{n+2}\right), \\
X_{n+3} & =(\delta+1)\left(\sum_{i=1}^{n} t_{i} \tilde{H}_{i}\right)+\frac{1}{2} \sum_{i=1}^{n+2} \sum_{j=1, j \neq i}^{n+2} C_{i j}-\theta_{n+2}\left(\rho+\theta_{n+2}+\theta_{n+3}\right) .
\end{aligned}
$$

Here we introduce the Hirota derivatives $D_{i}(i=1, \ldots, n)$ defined by

$$
P\left(D_{1}, \ldots, D_{n}\right) \varphi \cdot \psi=\left.P\left(\partial_{t_{1}}, \ldots, \partial_{t_{n}}\right)(\varphi(s+t) \psi(s-t))\right|_{t=0},
$$

where $P\left(D_{1}, \ldots, D_{n}\right)$ is a polynomial in the derivations $D_{i}(i=1, \ldots, n)$. By the definition, we obtain 


$$
\begin{aligned}
D_{i} \varphi \cdot \psi & =\partial_{t_{i}}(\varphi) \psi-\varphi \partial_{t_{i}}(\psi), \\
D_{i} D_{j} \varphi \cdot \psi & =\partial_{t_{i}} \partial_{t_{j}}(\varphi) \psi-\partial_{t_{i}}(\varphi) \partial_{t_{j}}(\psi)-\partial_{t_{j}}(\varphi) \partial_{t_{i}}(\psi)+\psi \partial_{t_{i}} \partial_{t_{j}}(\varphi)
\end{aligned}
$$

and

$$
\begin{aligned}
\partial_{t_{i}} \log \frac{\varphi}{\psi} & =\frac{D_{i} \varphi \cdot \psi}{\varphi \cdot \psi} \\
\partial_{t_{i}} \partial_{t_{j}} \log \varphi \psi & =\frac{D_{i} D_{j} \varphi \cdot \psi}{\varphi \cdot \psi}-\frac{D_{i} \varphi \cdot \psi}{\varphi \cdot \psi} \frac{D_{j} \varphi \cdot \psi}{\varphi \cdot \psi} .
\end{aligned}
$$

By substituting (3.25) into (3.35), we obtain the Toda and Toda-like equations expressed in terms of the Hirota derivatives.

Theorem 3.4. For the Schlesinger transformations $T_{k}(k=1, \ldots, n+3)$, we have the following Toda and Toda-like equations:

$$
\begin{aligned}
F_{k} T_{k}\left(\tau_{0}\right) T_{k}^{-1}\left(\tau_{0}\right)= & D_{k} D_{k+1} \tau_{0} \cdot \tau_{0}-\frac{2 C_{k k+1}}{\left(t_{k}-t_{k+1}\right)^{2}} \tau_{0}^{2} \quad(k=1, \ldots, n-1), \\
F_{n} T_{n}\left(\tau_{0}\right) T_{n}^{-1}\left(\tau_{0}\right)= & \sum_{i=1}^{n}\left(t_{i}-1\right) D_{i} D_{n} \tau_{0} \cdot \tau_{0}+2 \partial_{t_{n}}\left(\tau_{0}\right) \cdot \tau_{0}-\frac{2 C_{n n+1}}{t_{n}^{2}} \tau_{0}^{2}, \\
F_{n+1} T_{n+1}\left(\tau_{0}\right) T_{n+1}^{-1}\left(\tau_{0}\right)= & \sum_{i=1}^{n} \sum_{j=1}^{n}\left(t_{i}-1\right) t_{j} D_{i} D_{j} \tau_{0} \cdot \tau_{0} \\
& +2 \sum_{i=1}^{n}\left(2 t_{i}-1\right) \partial_{t_{i}}\left(\tau_{0}\right) \cdot \tau_{0}+2 C_{n+1 n+2} \tau_{0}^{2}, \\
F_{n+2} T_{n+2}\left(\tau_{0}\right) T_{n+2}^{-1}\left(\tau_{0}\right)= & \sum_{i=1}^{n} \sum_{j=1}^{n} t_{i}\left(t_{i}-1\right) t_{j} D_{i} D_{j} \tau_{0} \cdot \tau_{0}+2 \sum_{i=1}^{n} t_{i}^{2} \partial_{t_{i}}\left(\tau_{0}\right) \cdot \tau_{0} \\
& +2\left\{\theta_{n+2}\left(\rho+\theta_{n+2}\right)+\sum_{j=1}^{n+1} C_{i n+2}\right\} \tau_{0}^{2}, \\
& +2\left\{\theta_{n+2}\left(\rho+\theta_{n+2}+\theta_{n+3}\right)+\sum_{j=1}^{n+1} C_{i n+2}\right\} \tau_{0}^{2}, \\
F_{n+3} T_{n+3}\left(\tau_{0}\right) T_{n+3}^{-1}\left(\tau_{0}\right)= & \sum_{i=1}^{n} \sum_{j=1}^{n} t_{i}\left(t_{i}-1\right) t_{j} D_{i} D_{j} \tau_{0} \cdot \tau_{0}+2 \sum_{i=1}^{n} t_{i}^{2} \partial_{t_{i}}\left(\tau_{0}\right) \cdot \tau_{0} \\
&
\end{aligned}
$$

where 


$$
\begin{aligned}
F_{k}= & \left(t_{k}-t_{k+1}\right)^{-1 / 2} \prod_{j=1, j \neq k}^{n+2}\left(t_{k}-t_{j}\right)^{-\Gamma_{k}^{j}} \prod_{j=1, j \neq k+1}^{n+2}\left(t_{k+1}-t_{j}\right)^{-\Gamma_{-k+1}^{-j}} \\
& \times \prod_{i=1}^{n+2} \prod_{j=1, j \neq i}^{n+2}\left(t_{i}-t_{j}\right)^{-\Gamma_{k,-(k+1)} / 2} \quad(k=1, \ldots, n+1), \\
F_{n+2}= & \prod_{j=1}^{n+1}\left(t_{j}-1\right)^{-\Gamma_{n+2}^{j}} \prod_{i=1}^{n+2} \prod_{j=1, j \neq i}^{n+2}\left(t_{i}-t_{j}\right)^{-\Gamma_{n+2,-(n+3) / 2}}, \\
F_{n+3}= & \prod_{j=1}^{n+1}\left(t_{j}-1\right)^{-\Gamma_{n+2}^{j}} \prod_{i=1}^{n+2} \prod_{j=1, j \neq i}^{n+2}\left(t_{i}-t_{j}\right)^{-\Gamma_{n+2, n+3} / 2} .
\end{aligned}
$$

We note that the Toda equation for $T_{n+1}$ is equivalent to the equation given in [8].

\subsection{Hirota-Miwa equations}

In the following, we set

$$
\tau_{k, l}=T_{k, l}\left(\tau_{0}\right), \quad \tau_{k,-l}=T_{k,-l}\left(\tau_{0}\right) \quad(k, l=1, \ldots, n+3, k \neq l) .
$$

We first present the Hirota-Miwa equation for the following six $\tau$-functions:

$$
\tau_{n+2, n+3}, \quad \tau_{n+1, n+2}, \quad \tau_{n+2,-(n+1)}, \quad \tau_{n+1, n+3}, \quad \tau_{n+3,-(n+1)}, \quad \tau_{0} .
$$

The action of transformations $T_{n+1, n+2}, T_{n+3,-(n+1)}$ and $T_{n+2, n+3}$ on the Hamiltonians $H_{i}(i=1, \ldots, n)$ is described as follows:

$$
\begin{gathered}
T_{n+1, n+2}\left(H_{i}\right)=H_{i}-\frac{\operatorname{tr} A_{i} R_{n+1, n+2}}{t_{i}\left(t_{i}-1\right)}+\frac{\Gamma_{n+1}^{i}}{t_{i}}+\frac{\Gamma_{n+2}^{-i}}{t_{i}-1}+\sum_{j=1, j \neq i}^{n+2} \frac{\Gamma_{n+1, n+2}}{t_{i}-t_{j}}, \\
T_{n+3,-(n+1)}\left(H_{i}\right)=H_{i}+\frac{1}{t_{i}}\left(a_{i}-b_{i} \frac{a_{n+1}}{b_{n+1}}\right)+\frac{\Gamma_{-(n+1)}^{i}}{t_{i}}+\sum_{j=1, j \neq i}^{n+2} \frac{\Gamma_{n+3,-(n+1)}}{t_{i}-t_{j}}, \\
T_{n+2, n+3}\left(H_{i}\right)=H_{i}+\frac{1}{t_{i}-1}\left(a_{i}+b_{i} \frac{d_{n+2}}{b_{n+2}}\right)+\frac{\Gamma_{n+2}^{i}}{t_{i}-1}+\sum_{j=1, j \neq i}^{n+2} \frac{\Gamma_{n+2, n+3}}{t_{i}-t_{j}} .
\end{gathered}
$$

From (3.43) and

$$
\begin{array}{r}
d \log \tau_{k, l}=\sum_{i=1}^{n} T_{k, l}\left(H_{i}\right), \quad d \log \tau_{k,-l}=\sum_{i=1}^{n} T_{k,-l}\left(H_{i}\right) \\
(k, l=1, \ldots, n+3, k \neq l),
\end{array}
$$


we obtain

$$
\begin{aligned}
\frac{\tau_{n+1, n+2} \tau_{n+3,-(n+1)}}{\tau_{0} \tau_{n+2, n+3}}= & \left(d_{n+1}-b_{n+1} \frac{d_{n+2}}{b_{n+2}}\right) \\
& \times \prod_{i=1}^{n} t_{i}^{1 /(n+1)} \prod_{i=1}^{n+2} \prod_{j=1, j \neq i}^{n+2}\left(t_{i}-t_{j}\right)^{-1 /\{2(n+1)(n+2)\}} .
\end{aligned}
$$

Hence the Hirota-Miwa-equation

$$
\begin{aligned}
& \tau_{n+1, n+2} \tau_{n+3,-(n+1)}-\tau_{n+1, n+3} \tau_{n+2,-(n+1)} \\
& =\theta_{n+1} \prod_{i=1}^{n} t_{i}^{1 /(n+1)} \prod_{i=1}^{n+2} \prod_{j=1, j \neq i}^{n+2}\left(t_{i}-t_{j}\right)^{-1 /\{2(n+1)(n+2)\}} \tau_{0} \tau_{n+2, n+3}
\end{aligned}
$$

is obtained by the action of the transformation $r_{n+1}$ on the both sides of (3.45).

For the other indexies $i, j, k=1, \ldots, n+3$ with $i, j, k$ mutually distinct, the Hirota-Miwa equations are obtained in a similar way.

Theorem 3.5. For any distinct $i, j, k=1, \ldots, n+3$, we have the following Hirota-Miwa equations:

$$
F_{k}^{i j} \tau_{0} \tau_{i, j}=\tau_{i, k} \tau_{j,-k}-\tau_{j, k} \tau_{i,-k}
$$

where

$$
\begin{aligned}
F_{k}^{i j}= & \theta_{k}\left(t_{i}-t_{j}\right)^{1 / 2}\left(t_{i}-t_{k}\right)^{-1 / 2}\left(t_{j}-t_{k}\right)^{-1 / 2} \\
& \times \prod_{l=1, l \neq k}^{n+2}\left(t_{k}-t_{l}\right)^{1 /(n+1)} \prod_{l_{1}=1}^{n+2} \prod_{l_{2}=1, l_{2} \neq l_{1}}^{n+2}\left(t_{l_{1}}-t_{l_{2}}\right)^{-1 /\{2(n+1)(n+2)\}}, \\
F_{j}^{i, n+3}= & \theta_{j} \prod_{k=1, k \neq j}^{n+2}\left(t_{j}-t_{k}\right)^{1 /(n+1)} \prod_{k=1}^{n+2} \prod_{l=1, l \neq k}^{n+2}\left(t_{k}-t_{l}\right)^{-1 /\{2(n+1)(n+2)\}}, \\
F_{n+3}^{i j}= & \theta_{n+3}\left(t_{i}-t_{j}\right)^{1 / 2} \prod_{k=1}^{n+2} \prod_{l=1, l \neq k}^{n+2}\left(t_{k}-t_{l}\right)^{-1 /\{2(n+1)(n+2)\}} .
\end{aligned}
$$

\subsection{Bilinear differential equations}

In this section, we present the bilinear differential equations for the $\tau$ functions $\tau_{0}$ and $\tau_{1}=\tau_{n+1, n+2}$. We set

$$
\hat{H}_{i}=\sum_{j=1, j \neq i}^{n+2} \frac{t_{i}\left(t_{i}-1\right)}{t_{i}-t_{j}}\left(\operatorname{tr} A_{i} A_{j}-\frac{1}{2} \theta_{i} \theta_{j}\right) \quad(i=1, \ldots, n)
$$


and

$$
\hat{H}_{i}^{*}=T_{n+1, n+2}\left(\hat{H}_{i}\right)=\hat{H}_{i}-\operatorname{tr} A_{i} R_{n+1, n+2}+\frac{\theta_{i}}{2} \quad(i=1, \ldots, n) .
$$

Denoting $\hat{R}=R_{n+1, n+2}$, we have

$$
\partial_{t_{i}}(\hat{R})=\frac{\hat{R} A_{i}\left(\hat{R}-I_{2}\right)}{t_{i}-1}-\frac{\left(\hat{R}-I_{2}\right) A_{i} \hat{R}}{t_{i}} \quad(i=1, \ldots, n) .
$$

It follows that

$$
\begin{aligned}
\delta_{i}\left(\hat{H}_{i}\right) & =\sum_{j=1, j \neq i}^{n+2} \frac{t_{i}\left(t_{i}-1\right)\left(t_{i}^{2}-2 t_{i} t_{j}+t_{j}\right)}{\left(t_{i}-t_{j}\right)^{2}}\left(\operatorname{tr} A_{i} A_{j}-\frac{1}{2} \theta_{i} \theta_{j}\right), \\
\delta_{j}\left(\hat{H}_{i}\right)= & \frac{t_{i}\left(t_{i}-1\right) t_{j}\left(t_{j}-1\right)}{\left(t_{i}-t_{j}\right)^{2}}\left(\operatorname{tr} A_{i} A_{j}-\frac{1}{2} \theta_{i} \theta_{j}\right) \quad(j=1, \ldots, n, j \neq i), \\
\delta_{i}\left(\hat{H}_{i}-\hat{H}_{i}^{*}\right)= & \operatorname{tr} A_{i}\left(\hat{R}-I_{2}\right) A_{i} \hat{R}-\sum_{j=1, j \neq i}^{n+2} \frac{t_{i}\left(t_{i}-1\right)}{t_{i}-t_{j}} \operatorname{tr}\left[A_{i}, A_{j}\right] \hat{R}, \\
\delta_{j}\left(\hat{H}_{i}-\hat{H}_{i}^{*}\right)= & t_{j} \operatorname{tr} A_{i} \hat{R} A_{j}\left(\hat{R}-I_{2}\right)-\left(t_{j}-1\right) \operatorname{tr} A_{i}\left(\hat{R}-I_{2}\right) A_{j} \hat{R} \\
& -\frac{t_{j}\left(t_{j}-1\right)}{t_{i}-t_{j}} \operatorname{tr}\left[A_{i}, A_{j}\right] \hat{R} \quad(j=1, \ldots, n, j \neq i),
\end{aligned}
$$

where $\delta_{i}=t_{i}\left(t_{i}-1\right) \partial_{i}$, for each $i=1, \ldots, n$. By using (3.52), we obtain

$$
\begin{aligned}
\sum_{j=1}^{n} \frac{2}{2 t_{i} t_{j}-t_{i}-t_{j}}\left\{\delta_{j}\left(\hat{H}_{i}+\hat{H}_{i}^{*}\right)+\left(\hat{H}_{i}-\hat{H}_{i}^{*}\right)\left(\hat{H}_{j}-\hat{H}_{j}^{*}\right)\right\} \\
=-\frac{\operatorname{tr} A_{i}\left(\hat{R}-I_{2}\right) A_{i} \hat{R}}{t_{i}\left(t_{i}-1\right)}+\frac{1}{t_{i}\left(t_{i}-1\right)}\left(\operatorname{tr} A_{i} \hat{R}-\frac{\theta_{i}}{2}\right)^{2} \\
\quad+\sum_{j=1, j \neq i}^{n} \frac{2}{2 t_{i} t_{j}-t_{i}-t_{j}}\left\{\left(\operatorname{tr} A_{i} \hat{R}-\frac{\theta_{i}}{2}\right)\left(\operatorname{tr} A_{j} \hat{R}-\frac{\theta_{j}}{2}\right)\right. \\
\left.+\left(t_{j}-1\right) \operatorname{tr} A_{i}\left(\hat{R}-I_{2}\right) A_{j} \hat{R}-t_{j} \operatorname{tr} A_{i} \hat{R} A_{j}\left(\hat{R}-I_{2}\right)\right\} \\
\quad+\sum_{j=1, j \neq i}^{n+2} \frac{1}{2 t_{i} t_{j}-t_{i}-t_{j}}\left\{\left(2 t_{j}-1\right) \operatorname{tr}\left[A_{i}, A_{j}\right] \hat{R}-\operatorname{tr} A_{i} A_{j}+\frac{1}{2} \theta_{i} \theta_{j}\right\} \\
\quad+\sum_{j=1, j \neq i}^{n+2} \frac{2 t_{i}-1}{t_{i}-t_{j}}\left(\operatorname{tr} A_{i} A_{j}-\frac{1}{2} \theta_{i} \theta_{j}\right) \quad(i=1, \ldots, n) .
\end{aligned}
$$


On the other hand, we obtain

$$
\begin{aligned}
\operatorname{tr} A_{i}\left(\hat{R}-I_{2}\right) A_{j} \hat{R}= & \left(\operatorname{tr} A_{i} \hat{R}-\frac{\theta_{i}}{2}\right)\left(\operatorname{tr} A_{j} \hat{R}-\frac{\theta_{j}}{2}\right)-\frac{1}{2} \operatorname{tr}\left[A_{i}, A_{j}\right] \hat{R} \\
& -\frac{1}{2} \operatorname{tr} A_{i} A_{j}+\frac{1}{4} \theta_{i} \theta_{j} \quad(j=1, \ldots, n, j \neq i), \\
\operatorname{tr} A_{i} \hat{R} A_{j}\left(\hat{R}-I_{2}\right)= & \left(\operatorname{tr} A_{i} \hat{R}-\frac{\theta_{i}}{2}\right)\left(\operatorname{tr} A_{j} \hat{R}-\frac{\theta_{j}}{2}\right)+\frac{1}{2} \operatorname{tr}\left[A_{i}, A_{j}\right] \hat{R} \\
& -\frac{1}{2} \operatorname{tr} A_{i} A_{j}+\frac{1}{4} \theta_{i} \theta_{j} \quad(j=1, \ldots, n, j \neq i), \\
\operatorname{tr} A_{i}\left(\hat{R}-I_{2}\right) A_{i} \hat{R}= & \left(\operatorname{tr} A_{i} \hat{R}-\frac{\theta_{i}}{2}\right)^{2}-\frac{\theta_{i}^{2}}{4 t_{i}\left(t_{i}-1\right)}
\end{aligned}
$$

and

$$
\begin{aligned}
& \operatorname{tr}\left[A_{i}, A_{n+1}\right] \hat{R}+\operatorname{tr} A_{i} A_{n+1}=\theta_{n+1} \operatorname{tr} A_{i} \hat{R}, \\
& \operatorname{tr}\left[A_{i}, A_{n+2}\right] \hat{R}-\operatorname{tr} A_{i} A_{n+2}=\theta_{n+2} \operatorname{tr} A_{i}\left(\hat{R}-I_{2}\right)
\end{aligned}
$$

by direct computations for each $i=1, \ldots, n$. From (3.53), (3.54) and (3.55), the following differential equations are obtained:

$$
\begin{aligned}
\sum_{j=1}^{n} \frac{2}{2 t_{i} t_{j}-t_{i}-t_{j}}\left\{\delta_{j}\left(\hat{H}_{i}+\hat{H}_{i}^{*}\right)+\left(\hat{H}_{i}-\hat{H}_{i}^{*}\right)\left(\hat{H}_{j}-\hat{H}_{j}^{*}\right)\right\} \\
=\left(\frac{\theta_{n+1}}{t_{i}}+\frac{\theta_{n+2}}{t_{i}-1}\right)\left(\hat{H}_{i}-\hat{H}_{i}^{*}\right)+\frac{2 t_{i}-1}{t_{i}\left(t_{i}-1\right)} \hat{H}_{i}+\frac{\theta_{i}^{2}}{4 t_{i}\left(t_{i}-1\right)} \\
(i=1, \ldots, n) .
\end{aligned}
$$

By substituting

$$
\hat{H}_{i}=\delta_{i} \log \tau_{0}+\hat{C}_{i}, \quad \hat{H}_{i}^{*}=\delta_{i} \log \tau_{1}+\hat{C}_{i}^{*},
$$

where

$$
\hat{C}_{i}=\sum_{j=1, j \neq i}^{n+2} \frac{t_{i}\left(t_{i}-1\right)}{t_{i}-t_{j}}\left(C_{i j}+\frac{1}{2} \theta_{i} \theta_{j}\right), \quad \hat{C}_{i}^{*}=T_{n+1, n+2}\left(\hat{C}_{i}\right)
$$

into (3.56), we obtain the bilinear differential equations for the $\tau$-functions $\tau_{0}$ and $\tau_{1}$.

Theorem 3.6. The $\tau$-functions $\tau_{0}$ and $\tau_{1}$ satisfy the following bilinear differential equations: 


$$
\begin{gathered}
\sum_{j=1}^{n} \frac{2}{2 t_{i} t_{j}-t_{i}-t_{j}}\left\{D_{i}^{*} D_{j}^{*} \tau_{0} \cdot \tau_{1}+F_{j}^{i} D_{j}^{*} \tau_{0} \cdot \tau_{1}\right\}+F^{i, 0} D_{i}^{*} \tau_{0} \cdot \tau_{1} \\
-\frac{2 t_{i}-1}{t_{i}\left(t_{i}-1\right)} \delta_{i}\left(\tau_{0}\right) \cdot \tau_{1}+F^{i, 1} \tau_{0} \cdot \tau_{1}=0 \quad(i=1, \ldots, n),
\end{gathered}
$$

where

$$
\begin{aligned}
F_{j}^{i}= & \hat{C}_{i}-\hat{C}_{i}^{*} \\
F^{i, 0}= & \sum_{j=1}^{n} \frac{2\left(\hat{C}_{i}-\hat{C}_{i}^{*}\right)}{2 t_{i} t_{j}-t_{i}-t_{j}}-\frac{\theta_{n+1}}{t_{i}}-\frac{\theta_{n+2}}{t_{i}-1} \\
F^{i, 1}= & \sum_{j=1}^{n} \frac{2}{2 t_{i} t_{j}-t_{i}-t_{j}}\left\{\delta_{j}\left(\hat{C}_{i}+\hat{C}_{i}^{*}\right)+\left(\hat{C}_{i}-\hat{C}_{i}^{*}\right)\left(\hat{C}_{j}-\hat{C}_{j}^{*}\right)\right\} \\
& -\left(\frac{\theta_{n+1}}{t_{i}}+\frac{\theta_{n+2}}{t_{i}-1}\right)\left(\hat{C}_{i}-\hat{C}_{i}^{*}\right)-\frac{2 t_{i}-1}{t_{i}\left(t_{i}-1\right)} \hat{C}_{i}-\frac{\theta_{i}^{2}}{4 t_{i}\left(t_{i}-1\right)}
\end{aligned}
$$

and $D_{i}^{*}$ stands for the Hirota derivative with respect to the derivation $\delta_{i}$.

\section{Garnier system}

We consider rational functions in $a_{j}, b_{j}, c_{j}, d_{j}(j=1, \ldots, n+2)$ defined as

$$
\begin{array}{ll}
q_{i}=\frac{t_{i} b_{i}}{b_{\infty}} & (i=1, \ldots, n), \\
p_{i}=\frac{b_{\infty}}{t_{i}}\left\{\frac{a_{i}}{b_{i}}+\left(t_{i}-1\right) \frac{a_{n+1}}{b_{n+1}}-t_{i} \frac{a_{n+2}}{b_{n+2}}\right\} & (i=1, \ldots, n), \\
x_{i}=\frac{t_{i}}{t_{i}-1} & (i=1, \ldots, n),
\end{array}
$$

where $b_{\infty}=\sum_{j=1}^{n+2} t_{j} b_{j}$. Let $\{$,$\} be the Poisson bracket defined by$

$$
\{\varphi, \psi\}=\sum_{j=1}^{n}\left(\frac{\partial \varphi}{\partial p_{j}} \frac{\partial \psi}{\partial q_{j}}-\frac{\partial \varphi}{\partial q_{j}} \frac{\partial \psi}{\partial p_{j}}\right) .
$$

Also let $\bar{d}$ be an exterior differentiation with respect to $x_{1}, \ldots, x_{n}$. Then we have

Proposition 4.1 ([1]). The independent and dependent variables $q_{i}, p_{i}, x_{i}$ $(i=1, \ldots, n)$ defined by (4.1) satisfy the Garnier system 


$$
\bar{d} q_{i}=\sum_{j=1}^{n}\left\{\bar{H}_{j}, q_{i}\right\} d x_{j}, \quad \bar{d} p_{i}=\sum_{j=1}^{n}\left\{\bar{H}_{j}, p_{i}\right\} d x_{j}
$$

with the Hamiltonians

$$
-\left(x_{i}-1\right)^{2} \bar{H}_{i}=T_{n+3,-(n+1)}\left(H_{i}\right) \quad(i=1, \ldots, n) .
$$

Here we remark

$$
\bar{H}_{i}=K_{i}+\sum_{j=1, j \neq i}^{n+2} \frac{\bar{C}_{i j}}{x_{i}-x_{j}} \quad(i=1, \ldots, n),
$$

where

$$
\begin{aligned}
\bar{C}_{i j} & =T_{n+3,-(n+1)}\left(C_{i j}\right)+\theta_{i} \theta_{j} \quad(j=1, \ldots, n), \\
\bar{C}_{i n+1} & =T_{n+3,-(n+1)}\left(C_{i n+1}\right)+\theta_{i}\left(\theta_{n+1}-1\right), \\
\bar{C}_{i n+2} & =-\sum_{j=1, j \neq i}^{n+2} T_{n+3,-(n+1)}\left(C_{i j}\right)+\theta_{i}\left(\theta_{i}+\theta_{n+3}+2 \rho+1\right)
\end{aligned}
$$

and $K_{i}$ is given by (1.9).

In this section, we show that the Garnier system has affine Weyl group symmetry of type $B_{n+3}^{(1)}$. We also show that the $\tau$-functions for the Garnier system, formulated on the root lattice $Q\left(C_{n+3}\right)$, satisfy Toda equations, HirotaMiwa equations and bilinear differential equations.

\subsection{Affine Weyl group symmetries}

The transformations $\sigma_{k}, r_{l}$ and $T_{\mu}$ given in Section 2 can be lifted to the birational canonical transformations of the variables $q_{i}, p_{i}, x_{i}(i=1, \ldots, n)$ which is already known in [7,8]. In this section, we formulate the action of those transformations as realization of affine Weyl group.

Denote the parameter by

$$
\begin{aligned}
& \varepsilon_{1}=\theta_{n+1}, \quad \varepsilon_{2}=\theta_{n+2}, \quad \varepsilon_{3}=\theta_{n+3}+1, \\
& \varepsilon_{j}=\theta_{j-3} \quad(j=4, \ldots, n+3) .
\end{aligned}
$$

Then the group of symmetries for the Garnier system is generated by the transformations $s_{k}(k=0,1, \ldots, n+3)$ which act on $\varepsilon_{j}(j=1, \ldots, n+3)$ as follows: 
$(4.8)$

$$
\begin{array}{lrl}
s_{0}\left(\varepsilon_{1}\right)=1-\varepsilon_{2}, & s_{0}\left(\varepsilon_{2}\right)=1-\varepsilon_{1}, & s_{0}\left(\varepsilon_{j}\right)=\varepsilon_{j} \quad(j \neq 1,2), \\
s_{k}\left(\varepsilon_{j}\right)=\varepsilon_{\sigma_{k}(j)} & (k=1, \ldots, n+2), & s_{n+3}\left(\varepsilon_{j}\right)=(-1)^{\delta_{j n+3}} \varepsilon_{j} .
\end{array}
$$

We describe the action of $s_{k}$ on the variables $q_{i}, p_{i}, x_{i}(i=1, \ldots, n)$.

(4.9) $s_{0}\left(q_{j}\right)=\frac{p_{j}\left(q_{j} p_{j}-\varepsilon_{j+3}\right)}{Q_{1}\left(Q_{1}+\varepsilon_{3}\right)}, \quad s_{0}\left(q_{j} p_{j}\right)=\varepsilon_{j+3}-q_{j} p_{j}, \quad s_{0}\left(x_{i}\right)=\frac{1}{x_{i}}$

where

$$
Q_{1}=\sum_{l=1}^{n} q_{l} p_{l}+\frac{1}{2}\left(1-\sum_{l=1}^{n+3} \varepsilon_{l}\right)
$$

for $k=0$.

$$
s_{1}\left(q_{j}\right)=\frac{q_{j}}{x_{j}}, \quad s_{1}\left(p_{j}\right)=x_{j} p_{j}, \quad s_{1}\left(x_{i}\right)=\frac{1}{x_{i}}
$$

for $k=1$.

$$
s_{2}\left(q_{j}\right)=\frac{q_{j}}{Q_{2}}, \quad s_{2}\left(p_{j}\right)=\left(p_{j}-Q_{1}\right) Q_{2}, \quad s_{2}\left(x_{i}\right)=\frac{x_{i}}{x_{i}-1}
$$

where

$$
Q_{2}=\sum_{j=1}^{n} q_{j}-1
$$

for $k=2$.

$$
\begin{aligned}
& s_{3}\left(q_{1}\right)=\frac{1}{q_{1}}, \quad s_{3}\left(q_{j}\right)=-\frac{q_{j}}{q_{1}} \quad(j \neq 1), \\
& s_{3}\left(p_{1}\right)=-q_{1} Q_{1}, \quad s_{3}\left(p_{j}\right)=-q_{1} p_{j} \quad(j \neq 1), \\
& s_{3}\left(x_{1}\right)=\frac{1}{x_{1}}, \quad s_{n}\left(x_{i}\right)=\frac{x_{i}}{x_{1}} \quad(i \neq 1)
\end{aligned}
$$

for $k=3$.

$$
s_{k}\left(q_{j}\right)=q_{\sigma_{k-3}(j)}, \quad p_{k}\left(q_{j}\right)=p_{\sigma_{k-3}(j)}, \quad s_{k}\left(x_{i}\right)=x_{\sigma_{k-3}(i)}
$$

for $k=4, \ldots, n+2$. 


$$
\begin{aligned}
& s_{n+3}\left(q_{j}\right)=q_{j}, \\
& s_{n+3}\left(p_{n}\right)=p_{n}-\frac{\varepsilon_{n+3}}{q_{n}}, \quad s_{n+3}\left(p_{j}\right)=p_{j} \quad(j \neq n), \\
& s_{n+3}\left(x_{i}\right)=x_{i}
\end{aligned}
$$

for $k=n+3$. The group generated by these $s_{k}$ is isomorphic to affine Weyl group $W\left(B_{n+3}^{(1)}\right)$.

Theorem 4.2. The birational canonical transformations $s_{k}(k=0, \ldots, n+3)$ satisfy the fundamental relations for the generators of $W\left(B_{n+3}^{(1)}\right)$

$$
\begin{array}{ll}
s_{k}^{2}=1 & (k=0, \ldots, n+3), \\
\left(s_{k} s_{l}\right)^{2}=1 & (k, l \neq 0,1,2,|k-l|>1), \\
\left(s_{k} s_{k+1}\right)^{3}=1 & (k=1, \ldots, n+1), \\
\left(s_{0} s_{1}\right)^{2}=1, & \left(s_{0} s_{2}\right)^{3}=1, \quad\left(s_{n+2} s_{n+3}\right)^{4}=1 .
\end{array}
$$

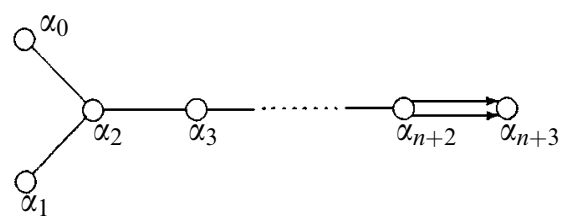

Fig. 1. Dynkin diagram of type $B_{n+3}^{(1)}$

The simple affine roots of $B_{n+3}^{(1)}$ is given as

$$
\begin{aligned}
& \alpha_{0}=1-\varepsilon_{1}-\varepsilon_{2}, \\
& \alpha_{j}=\varepsilon_{j}-\varepsilon_{j+1} \quad(j=1, \ldots, n+2), \\
& \alpha_{n+3}=\varepsilon_{n+3}
\end{aligned}
$$

and the action of $s_{k}$ on $\alpha_{j}(j=0,1, \ldots, n+3)$ is described as follows.

$$
s_{0}\left(\alpha_{0}\right)=-\alpha_{0}, \quad s_{0}\left(\alpha_{2}\right)=\alpha_{0}+\alpha_{2}, \quad s_{0}\left(\alpha_{j}\right)=\alpha_{j} \quad(j \neq 0,2)
$$

for $k=0$.

$$
s_{1}\left(\alpha_{1}\right)=-\alpha_{1}, \quad s_{1}\left(\alpha_{2}\right)=\alpha_{1}+\alpha_{2}, \quad s_{1}\left(\alpha_{j}\right)=\alpha_{j} \quad(j \neq 0,1)
$$

for $k=1$. 


$$
\begin{aligned}
& s_{2}\left(\alpha_{2}\right)=-\alpha_{2}, \\
& s_{2}\left(\alpha_{j}\right)=\alpha_{j}+\alpha_{2} \quad(j=0,1,3), \\
& s_{2}\left(\alpha_{j}\right)=\alpha_{j} \quad(j \neq 0,1,2,3)
\end{aligned}
$$

for $k=2$.

$$
\begin{aligned}
& s_{k}\left(\alpha_{k}\right)=-\alpha_{k}, \quad s_{k}\left(\alpha_{k+1}\right)=\alpha_{k+1}+\alpha_{k}, \quad s_{k}\left(\alpha_{k-1}\right)=\alpha_{k-1}+\alpha_{k}, \\
& s_{k}\left(\alpha_{j}\right)=\alpha_{j} \quad(j \neq k, k+1, k-1)
\end{aligned}
$$

for $k=3, \ldots, n+2$.

$$
\begin{aligned}
s_{n+3}\left(\alpha_{n+3}\right) & =-\alpha_{n+3}, \quad s_{n+3}\left(\alpha_{n+2}\right)=\alpha_{n+2}+2 \alpha_{n+3}, \\
s_{n+3}\left(\alpha_{j}\right) & =\alpha_{j} \quad(j \neq n+2, n+3)
\end{aligned}
$$

for $k=n+3$.

Remark 4.3. The group generated by the transformations $s_{1}, \ldots, s_{n+2}$ is isomorphic to the symmetric group $\Xi_{n+3}[1]$. Furthermore, the group generated by $s_{1}, \ldots, s_{n+3}$ is isomorphic to $W\left(B_{n+3}\right)$; e.g. [5].

Remark 4.4. In the only case $n=1$, there is the following birational canonical transformation:

$$
\begin{aligned}
& s_{0}^{*}(q)=q-\frac{\varepsilon_{4}}{p}, \quad s_{0}^{*}(p)=p, \quad s_{0}^{*}(t)=t, \\
& s_{0}^{*}\left(\varepsilon_{j}\right)=\varepsilon_{j}+\frac{1}{2}\left(1-\varepsilon_{1}-\varepsilon_{2}-\varepsilon_{3}-\varepsilon_{4}\right) \quad(j=1, \ldots, 4) .
\end{aligned}
$$

The transformation $s_{0}$ is generated by a composition of $s_{0}^{*}$ and $s_{1}, \ldots, s_{4}$.

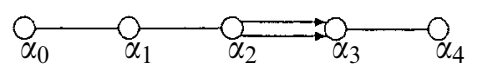

Fig. 2. Dynkin diagram of type $F_{4}^{(1)}$

But $s_{0}^{*}$ cannot be generated by a composition of $s_{0}, s_{1}, \ldots, s_{4}$. It follows that the group of symmetries for the Garnier system in 1-variable contains affine Weyl group $W\left(B_{4}^{(1)}\right)$. Actually, it is known that $P_{V I}$ has affine Weyl group symmetry of type $F_{4}^{(1)}$. The simple affine roots of $F_{4}^{(1)}$ is given by

$$
\begin{aligned}
& \alpha_{0}=\varepsilon_{1}-\varepsilon_{2}, \quad \alpha_{1}=\varepsilon_{2}-\varepsilon_{3}, \quad \alpha_{2}=\varepsilon_{3}-\varepsilon_{4}, \\
& \alpha_{3}=\varepsilon_{4}, \quad \alpha_{4}=\frac{1}{2}\left(1-\varepsilon_{1}-\varepsilon_{2}-\varepsilon_{3}-\varepsilon_{4}\right)
\end{aligned}
$$


and $s_{0}^{*}, s_{1}, \ldots, s_{4}$ act on $\alpha_{j}(j=0,1, \ldots, 4)$ as follows:

$$
\begin{aligned}
& s_{0}^{*}\left(\alpha_{4}\right)=-\alpha_{4}, \quad s_{0}^{*}\left(\alpha_{3}\right)=\alpha_{3}+\alpha_{4}, \quad s_{0}^{*}\left(\alpha_{j}\right)=\alpha_{j} \quad(j \neq 3,4), \\
& s_{1}\left(\alpha_{0}\right)=-\alpha_{0}, \quad s_{1}\left(\alpha_{1}\right)=\alpha_{1}+\alpha_{0}, \quad s_{1}\left(\alpha_{j}\right)=\alpha_{j} \quad(j \neq 0,1), \\
& s_{2}\left(\alpha_{1}\right)=-\alpha_{1}, \quad s_{2}\left(\alpha_{i}\right)=\alpha_{i}+\alpha_{1}, \quad s_{2}\left(\alpha_{j}\right)=\alpha_{j} \quad(i=0,2, j=3,4), \\
& s_{3}\left(\alpha_{2}\right)=-\alpha_{2}, \quad s_{3}\left(\alpha_{i}\right)=\alpha_{i}+\alpha_{2}, \quad s_{3}\left(\alpha_{j}\right)=\alpha_{j} \quad(i=1,3, j=0,4), \\
& s_{4}\left(\alpha_{3}\right)=-\alpha_{3}, \quad s_{4}\left(\alpha_{2}\right)=\alpha_{2}+2 \alpha_{3}, \quad s_{4}\left(\alpha_{4}\right)=\alpha_{4}+\alpha_{3}, \\
& s_{4}\left(\alpha_{j}\right)=\alpha_{j} \quad(j=0,1) .
\end{aligned}
$$

\section{2. $\tau$-Functions}

For each solution of the Garnier system, we introduce the $\tau$-functions $\bar{\tau}_{\mu}(\mu \in L)$ satisfying the Pfaffian systems

$$
\bar{d} \log \bar{\tau}_{\mu}=\sum_{i=1}^{n} T_{\mu}\left(\bar{H}_{i}\right) d x_{i} .
$$

Each $\bar{\tau}_{\mu}$ is determined up to multiplicative constants. From (4.4), we can identify these $\bar{\tau}_{\mu}$ with the $\tau$-functions for the Schlesinger system by

$$
\bar{\tau}_{0}=\tau_{n+3,-(n+1)} .
$$

Hence we can apply the properties of the $\tau$-functions $\tau_{\mu}$ system to the Garnier system. For each $\mu \in L$, the action of the birational canonical transformations $s_{k}$ on $\bar{\tau}_{\mu}$ is defined by

$$
s_{k}\left(\bar{\tau}_{\mu}\right)=\bar{\tau}_{s_{k}(\mu)} \quad(k=0,1, \ldots, n+3),
$$

where

$$
\begin{aligned}
s_{0}(\mu) & =\left(1-\mu_{2}, 1-\mu_{1}, \mu_{3}, \ldots, \mu_{n+3}\right), \\
s_{k}(\mu) & =\left(\mu_{(k, k+1) 1}, \ldots, \mu_{(k, k+1)(n+3)}\right) \quad(k=1, \ldots, n+2), \\
s_{n+3}(\mu) & =\left(\mu_{1}, \ldots, \mu_{n+2},-\mu_{n+3}\right)
\end{aligned}
$$

and $(k, k+1)$ stands for the adjacent transpositions. We also obtain bilinear relations which are satisfied by $\bar{\tau}_{\mu}$ formulated on the root lattice $Q\left(C_{n+3}\right)$.

Theorem 4.5. The $\tau$-functions $\bar{\tau}_{\mu}(\mu \in L)$ satisfy the Toda equations, the Hirota-Miwa equations and the bilinear differential equations given in Section 3.

In the last, we present the following proposition. 
Proposition 4.6. For the $\tau$-functions

$$
\bar{\tau}_{1,-2}=\bar{\tau}_{\mathbf{e}_{1}-\mathbf{e}_{2}}, \quad \bar{\tau}_{1,3}=\bar{\tau}_{\mathbf{e}_{1}+\mathbf{e}_{3}}, \quad \bar{\tau}_{1,-3}=\bar{\tau}_{\mathbf{e}_{1}-\mathbf{e}_{3}}
$$

and $\bar{\tau}_{0}$, the following relations are satisfied:

$$
\begin{aligned}
q_{i} & =-\frac{1}{\varepsilon_{3}} x_{i}\left(x_{i}-1\right) \frac{\partial}{\partial x_{i}} \log \frac{\bar{\tau}_{1,3}}{\bar{\tau}_{1,-3}}+2 \bar{X}_{i} \quad(i=i, \ldots, n), \\
q_{i} p_{i} & =-x_{i} \frac{\partial}{\partial x_{i}} \log \frac{\bar{\tau}_{1,-2}}{\bar{\tau}_{0}}+\frac{\bar{\Gamma}_{-1}^{j+3}-x_{i} \bar{\Gamma}_{-2}^{j+3}-\left(\varepsilon_{1}-\varepsilon_{2}\right) \bar{X}_{i}}{x_{i}-1} \quad(i=1, \ldots, n),
\end{aligned}
$$

where

$$
\bar{X}_{i}=\sum_{j=1, j \neq i}^{n+2} \frac{x_{i}\left(x_{j}-1\right)}{(n+1)(n+2)\left(x_{i}-x_{j}\right)}, \quad \bar{\Gamma}_{-k}^{i}=-\frac{\varepsilon_{i}}{2}+\frac{1-2 \varepsilon_{k}}{2(n+1)} .
$$

Proof. By using (4.1), (4.7) and (4.28), we can rewrite the relations (4.31) into

$$
\begin{aligned}
q_{i}= & \frac{t_{i}}{\theta_{n+3}+1} \partial_{i} \log \frac{\tau_{2 \mathbf{e}_{n+3}}}{\tau_{0}}-\sum_{j=1, j \neq i}^{n+2} \frac{2 t_{i}}{(n+1)(n+2)\left(t_{i}-t_{j}\right)}, \\
q_{i} p_{i}= & t_{i}\left(t_{i}-1\right) \partial_{i} \log \frac{\tau_{n+3,-(n+2)}}{\tau_{n+3,-(n+1)}}+\left(t_{i}-1\right) \Gamma_{-(n+1)}^{i}-t_{i} \Gamma_{-(n+2)}^{i} \\
& +\sum_{j=1, j \neq i}^{n+2} \frac{t_{i}\left(t_{i}-1\right)\left(\theta_{n+1}-\theta_{n+2}\right)}{(n+1)(n+2)\left(t_{i}-t_{j}\right)} \quad(i=1, \ldots, n),
\end{aligned}
$$

where

$$
\Gamma_{-k}^{i}=-\frac{\theta_{i}}{2}+\frac{1-2 \theta_{k}}{2(n+1)}
$$

Hence we show the relations (4.33) in the following.

We consider the Schlesinger transformations $T_{2 \mathbf{e}_{n+3}}$ which act on the parameters as follows:

$$
T_{2 \mathbf{e}_{n+3}}\left(\theta_{j}\right)=\theta_{j}+2 \delta_{j n+3} \quad(j=1, \ldots, n+3) .
$$

The action of $T_{2 \mathbf{e}_{n+3}}$ on the Hamiltonians $H_{i}(i=1, \ldots, n)$ is described as follows:

$$
T_{2 \mathbf{e}_{n+3}}\left(H_{i}\right)=H_{i}+\left(\theta_{n+3}+1\right) \frac{b_{i}}{b_{\infty}}+\sum_{j=1, j \neq i}^{n+2} \frac{2\left(\theta_{n+3}+1\right)}{(n+1)(n+2)\left(t_{i}-t_{j}\right)} .
$$


From (3.44) and (4.36), the first relation of (4.33) is obtained. The second relation of (4.33) is obtained in a similar way.

Acknowledgement. The auther is grateful to Professors Masatoshi Noumi, Masa-Hiko Saito and Yasuhiko Yamada for valuable discussions and advices.

\title{
References
}

[1] Iwasaki, K., Kimura, H., Shimomura, S., and Yoshida, M., From Gauss to Painlevé-A Modern Theory of Special Functions, Aspects of Mathematics, E16 (1991).

[2] Jimbo, M., Miwa, T., and Ueno, K., Monodromy preserving deformation of linear ordinary differential equations with rational coefficients I, Physica, 2D (1981), 306-352.

[ 3 ] Jimbo, M. and Miwa, T., Monodromy preserving deformation of linear ordinary differential equations with rational coefficients II, Physica, 2D (1981), 407-448.

[4] Masuda, T., On a class of algebraic solutions to the Painlevé VI equation, its determinant formula and coalescence cascade, Funkcial. Ekvac., 46 (2003), 121-171.

[ 5] Okamoto, K., Studies on the Painlevé equations, I, Ann. Math. Pura Appl., 146 (1987), $337-381$.

[6] Okamoto, K., The Hamiltonians associated with the Painlevé equations, The Painlevé property: One Century Later, ed. Conte, R., CRM Series in Mathematical Physics, Springer, 1999.

[ 7 ] Tsuda, T., Birational symmetries, Hirota bilinear forms and special solutions of the Garnier systems in 2-variables, J. Math. Sci. Univ. Tokyo, 10 (2003), 355-371.

[ 8 ] Tsuda, T., Rational solutions of the Garnier system in terms of Schur polynomials, Int. Math. Res. Not., 43 (2003), 2341-2358.

\author{
nuna adreso: \\ Graduate School of Science and Technology \\ Kobe University \\ Rokko, Kobe 657-8501 \\ Japan \\ E-mail: suzukit@math.sci.kobe-u.ac.jp
}

(Ricevita la 25-an de decembro, 2003)

(Reviziita la 16-an de februaro, 2005) 